\title{
ON HEAT KERNEL DECAY \\ FOR THE RANDOM CONDUCTANCE MODEL
}

\author{
OMAR BOUKHADRA
}

To the innocence of Timy and Kus

Department of Mathematics, University of Constantine 1

boukhadra@umc.edu.dz

\begin{abstract}
We study discrete time random walks in an environment of i.i.d. nonnegative bounded conductances in $\mathbb{Z}^{d}$. We are interested in the anomaly of the heat kernel decay. We improve recent results and techniques.

keywords : Markov chains, Random walk, Random environments, Random conductances, Percolation.
\end{abstract}

AMS 2000 Subject Classification : 60G50; 60J10; 60K3\%.

\section{IntRoduCtion AND STATEMENT OF THE RESUltS}

The present work focuses on the Random Conductance Model (RCM) with i.i.d. conductances on the grid $\mathbb{Z}^{d}$. Explicitly, let $E_{d}$ be the set of non oriented nearest-neighbor bonds, i.e. $E_{d}=\left\{e=\{x, y\}: x, y \in \mathbb{Z}^{d},|x-y|=1\right\}$. Let $\left(\omega_{e}\right)_{e \in E_{d}}$ be a family of i.i.d. (non-negative) conductances defined on a probability space $(\Omega, \mathbb{P})$. The expectation with respect to $\mathbb{P}$ is denoted by $\mathbb{E}$. We write $\omega_{e}=\omega_{x y}=\omega(x, y)$ and $x \sim y$ if $\{x, y\} \in E_{d}$. For any realization of the environment $\omega \in \Omega$, set

$$
\pi(x)=\sum_{y:|x-y|=1} \omega_{x y}, \quad \mathrm{P}_{\omega}(x, y)=\frac{\omega_{x y}}{\pi(x)}, \quad \pi(A)=\sum_{x \in A} \pi(x) .
$$

With these settings, we associate $X=\left(X_{n}\right)$ the Markov chain with state-space $\mathbb{Z}^{d}$ and transition probability $\mathrm{P}_{\omega}(x, y)$ and let $P_{\omega}^{x}$ denote the quenched law of $X$, started from $x$ with

$$
P_{\omega}^{x}\left(X_{0}=x\right)=1
$$

Let $\mathrm{P}_{\omega}^{n}$ denote the $n$-th power of the transition kernel $P_{\omega}$, i.e.,

$$
\mathrm{P}_{\omega}^{n}(x, y)=P_{\omega}^{x}\left(X_{n}=y\right) .
$$

$X$ is then a random walk in the random environment $\omega$.

Assume that $\mathbb{P}\left(\omega_{e}>0\right)>p_{c}(d)$ where $p_{c}(d)$ is the critical value for Bernoulli bond percolation on $\mathbb{Z}^{d}$, and let $\mathscr{C}$ be the infinite cluster where bonds are open if they have positive conductances (see [6]). When $\mathbb{P}\left(\omega_{e}>0\right)$ is sufficiently close to one, the set $\mathbb{Z}^{d} \backslash \mathscr{C}$ is a union of finite clusters. 
Let $o \in \mathbb{Z}^{d}$ denote the origin and define the conditional measure

$$
\mathbb{P}_{o}(\cdot):=\mathbb{P}(\cdot \mid o \in \mathscr{C}) .
$$

The RCM model (see [7]) has witnessed an intense research activity in recent years. The particularity of the RCM is what we call anomalous behavior of the probability of return in the sense that its decay at time $2 n$ is slower than the standard one, i.e. slower than $n^{-d / 2}$. This phenomenon that depends on the environment law $\mathbb{P}$ was first observed and investigated for the case of annealed (averaged on the environment) return probabilities in Fontes and Mathieu [5]. The reason for such behavior for a random walk among random conductances is the trapping effect of clusters of small conductances in the environment (these clusters are often referred to as holes). Then, for the quenched $(\mathbb{P}$-a.s.) case, we have [1] where it was proved anomalous decay for $d \geq 5$ for heavy-tailed conductances. Afterwards, in [3]-[4], it has been shown that the transition from a normal decay with rate $n^{-d / 2}$ to a slower decay happens in the class of power tail laws of the environment. As for $d=4$, there is still anomality which has been established in [2].

In the present work, our main result is the following which basically reconsiders and improves the result and techniques in [3].

Theorem 1.1 Let $d \geq 2$ and $\alpha \in(0,1 / 2)$. Suppose that the environment is governed by bounded i.i.d. random conductances such that $\mathbb{P}\left(\omega_{e} \in[0,1]\right)=1$ and

$$
(4 d-2) \lim _{u \rightarrow 0} \frac{\log \mathbb{P}\left(\omega_{b} \in[u, 2 u]\right)}{\log u}<\alpha,
$$

Then, there exists $c>0$, such that $\mathbb{P}_{o}$-a.s. for any $n$ large enough, we have

$$
\mathrm{P}_{\omega}^{2 n}(o, o) \geq c \pi(o) n^{-(2+\alpha(d-1))}
$$

Remarks 1.2 (1) The lower bound (1.5) is to be compared with general upper bounds from [1, Theorem 2.1], and the general lower bound $\mathrm{P}_{\omega}^{2 n}(o, o) \geq c n^{-d / 2}$ (see for example [3, Remark 1.3]). Then we observe that we always have a normal decay in $d=2,3$ and our lower bound (1.5) is interesting for $d \geq 5$ and

$$
\alpha<\frac{1}{2} \frac{d-4}{d-1}
$$

(2) In the special case with i.i.d. polynomial lower tail conductances, i.e.

$$
\mathbb{P}\left(\omega_{e}<u\right)=u^{\gamma}(1+o(1)), \quad u \rightarrow 0,
$$

one can easily see that the condition (C) becomes $\gamma<\alpha /(4 d-2)$. This last condition on $\gamma$ and the estimate (1.5) yield

$$
\liminf _{n} \frac{\log \mathrm{P}_{\omega}^{2 n}(o, o)}{\log n} \geq-2-(d-1)(4 d-2) \gamma .
$$




\section{Proof of Theorem 1.1}

Henceforth, we let $d \geq 2$ and assume that the conductances $\left\{\omega_{e}, e \in E_{d}\right\}$ are i.i.d. random variables with $\omega_{e} \in[0,1]$ a.s. and $\mathbb{P}\left(\omega_{e}>0\right)>p_{c}(d)$. Let $\mathscr{C}$ denote the infinite cluster along positive conductances. (See $[6]$ ).

Henceforth, we call a box centered at the origin $B_{r}$ of radius $r$ on $\mathbb{Z}^{d}$, the set

$$
B_{r}=[-r, r]^{d} \cap \mathbb{Z}^{d},
$$

and $\partial B_{r}$ the frontier of $B_{r}$, that is, the sites of $B_{r}$ with a neighbor outside $B_{r}$.

Now pick a $\xi>0$ such that $\mathbb{P}\left(\omega_{e} \geq \xi\right)>p_{c}(d)$. There exists then almost surely a unique infinite cluster of bonds with conductances larger than $\xi$ that we denote by $\mathscr{C}_{\xi}$. It also represents the set of all sites in $\mathbb{Z}^{d}$ that have a path to infinity along edges with conductances at least $\xi$. Note that $\mathscr{C}_{\xi} \subset \mathscr{C}$. When $\xi$ is small enough, the components of $\mathscr{C} \backslash \mathscr{C}_{\xi}$ that we call holes are finite a.s. and in a box of radius $n$, they have logarithmic size (see [4, Lemma 4.1]). For $x \in \mathscr{C}$, call $\mathscr{H}_{x}$ be the set of vertices in the finite component of $\mathscr{C} \backslash \mathscr{C}_{\xi}$ containing $x$. The condition $(\mathrm{C})$ supposes that the conductances law is heavy tailed and hence $\bigcup_{\xi>0} \mathscr{C}_{\xi}=\mathbb{Z}^{d}$.

We are now interested in the expected time spent by the random walk in a box $B_{r}$. First, we have to consider the so called coarse-grained walk $X^{\xi}$ which records the successive visits of $X$ to $\mathscr{C}_{\xi}$. Set $T_{0}:=0$ and define

$$
T_{\ell+1}:=\inf \left\{n>T_{0}+\ldots+T_{\ell}: X_{n} \in \mathscr{C}_{\xi}\right\}-\left(T_{0}+\ldots+T_{\ell}\right), \quad \ell \geq 1 .
$$

For $\xi$ small enough, all components of $\mathscr{C} \backslash \mathscr{C}_{\xi}$ are finite a.s., and then $T_{\ell}<\infty$ a.s. for all $\ell$. These visits occur at the locations

$$
X_{\ell}^{\xi}:=X_{T_{0}+\cdots+T_{\ell}} .
$$

The sequence $\left(X_{n}^{\xi}\right)$ is a Markov chain on $\mathscr{C}_{\xi}$ with transition kernel given by

$$
\mathrm{P}_{\xi}(x, y):=P_{\omega}^{x}\left(X_{T_{1}}=y\right) .
$$

We easily check that the restriction of the measure $\pi$ to $\mathscr{C}_{\xi}$ is invariant and reversible for the Markov chain on $\mathscr{C}_{\xi}$ induced by $\mathrm{P}_{\xi}$. Set $\hat{\tau}_{r}=\inf \left\{\ell \geq 0: X_{\ell}^{\xi} \notin B_{r}\right\}$.

Lemma 2.1 For almost every $\omega \in\{o \in \mathscr{C}\}$, for $\xi$ small enough, there exists $c=$ $c(d, \xi)<\infty$ such that for any $r$ large enough,

$$
E_{\omega}^{o}\left(\hat{\tau}_{r}\right) \leq c r^{2}
$$

Proof. Let $r>0$ and set $n=c_{*} r^{2}$ where $c_{*}>0$ is chosen later. Choose $\xi$ small enough such that $\left\{o \in \mathscr{C}_{\xi}\right\}$ and [4, Lemma 4.1] holds. From [1, Lemma 3.2], the return probabilities decay of $X^{\xi}$ is standard. Thus for $x \in \mathscr{C}_{\xi} \cap B_{r}$, we have for $n$ large enough

$$
P_{\omega}^{x}\left(X_{n}^{\xi} \in B_{r}\right) \leq \sum_{y \in \mathscr{C}_{\xi} \cap B_{r}} \mathrm{P}_{\xi}^{n}(x, y) \leq c_{1} c_{2} n^{-d / 2} r^{d} \leq \frac{1}{2},
$$


where we choose $c_{*} \geq\left(2 c_{1} c_{2}\right)^{2 / d}$. Hence, $P_{\omega}^{x}\left(\hat{\tau}_{r}>n\right) \leq 1 / 2$, and the Markov property then gives, for $k$ a positive integer,

$$
P_{\omega}^{o}\left(\hat{\tau}_{r}>(k+1) n\right) \leq P_{\omega}^{o}\left(P_{\omega}^{X_{k n}^{\xi}}\left(\hat{\tau}_{r}>n\right) ; \hat{\tau}_{r}>k n\right) \leq \frac{1}{2} P_{\omega}^{o}\left(\hat{\tau}_{r}>k n\right) .
$$

Hence by induction, we have $P_{\omega}^{o}\left(\hat{\tau}_{r}>k n\right) \leq 1 / 2^{k}$, which yields

$$
E_{\omega}^{o}\left(\hat{\tau}_{r}\right)=\sum_{k \geq 0} E_{\omega}^{o}\left(\hat{\tau}_{r} ; k n<\hat{\tau}_{r} \leq(k+1) n\right) \leq n \sum_{k} \frac{k+1}{2^{k}} \leq c n
$$

Recall $\mathscr{H}_{y}$. Then, for any $x \in \mathscr{C}_{\xi}$, set

$$
\mathscr{F}_{x}=\bigcup_{y: \omega_{x y}>0} \mathscr{H}_{y}, \quad \mathscr{F}_{x}^{\prime}=\{x\} \cup \mathscr{F}_{x}
$$

and let

$$
\mathscr{G}_{x}=\mathscr{F}_{x}^{\prime} \bigcup \bigcup_{y \in \mathscr{b}_{\xi}, y \sim x} \mathscr{F}_{y}^{\prime} .
$$

Note that $\mathscr{G}_{x}$ is the set of sites where $X$ can be hidden before stepping again onto the strong component $\mathscr{C}_{\xi}$. Then, for $H_{r}=\inf \left\{k \geq 0: X_{k} \in \partial B_{r}\right\}$ we have:

Lemma 2.2 For $\mathbb{P}$-a.e. $\omega \in\{o \in \mathscr{C}\}$, for $\xi>0$ small enough, there exists $c=c(d, \xi)<$ $\infty$ such that for $r$ large enough, we have

$$
E_{\omega}^{o}\left(H_{r}\right) \leq c r^{2} \sup _{x \in \mathscr{C}_{\xi} \cap B_{r}}\left|\mathscr{G}_{x}\right| .
$$

Proof. Choose $\xi$ small enough such that Lemma 2.1 holds. Then, observe that

$$
H_{r} \leq \tau_{r} \leq \sum_{\ell=0}^{\hat{\tau}_{r}} T_{\ell}
$$

By the Markov property, we obtain therefore

$$
E_{\omega}^{o}\left(\sum_{\ell=0}^{\hat{\tau}_{r}} T_{\ell}\right) \leq E_{\omega}^{o}\left(\sum_{\ell=0}^{\hat{\tau}_{r}} E^{X_{\ell}^{\xi}}\left(T_{1}\right)\right) \leq \sup _{x \in \mathscr{C}_{\xi} \cap B_{r}} E^{x}\left(T_{1}\right) E_{\omega}^{o}\left(\hat{\tau}_{r}\right)
$$

Then use Lemma 2.1 and [1, Lemma 3.8] to get the desired estimate.

For the following, we introduce a special configuration of the environment that we call trap. Explicitly, let $\mathcal{A}_{n}$ be the event in the environment defined as follows: an edge $e=\{y, z\}$ with conductance $\omega_{e} \geq c>0$, which we call the strong edge, is such that for any $e^{\prime} \neq e$ incident with either $y$ or $z, \omega_{e^{\prime}} \in[1 / n, 2 / n]$. The configuration $\mathcal{A}_{n}$ is then made up of a strong edge and of $4 d-2$ weak edges with conductances of order $1 / n$. Let $\mathcal{A}_{n}(x)$ be the event on the space of environments that $x$ is a vertex neighboring a trap edge, which trap is situated outside the hypercubic box $B_{\max x_{i}}$, where the $x_{i}, i=1, \ldots, d$ are the associated coordinates of $x$. We say that the walk will meet a trap $\mathcal{A}_{n}$ at a time $k$ if we have $\mathcal{A}_{n}\left(X_{k}\right)$. 
As we proceeded in recent works (see [3]-[2]), to get the anomalous lower bound (1.5), we let the walk fall into a trap. The following lemma proves that the walk before exiting a box $B_{n^{\alpha}}$ will meet a.s. a trap $\mathcal{A}_{n}$.

Lemma 2.3 Suppose that the condition (C) holds and set $r=n^{\alpha}$. Then, there exist constants $c, \beta$ such that for $\mathbb{P}$-a.e $\omega \in\{o \in \mathscr{C}\}$, there exists $N=N(\omega)<\infty$ a.s. such that for all $n \geq N$,

$$
P_{\omega}^{o}\left(\bigcap_{k=0}^{r / 3-1} \mathcal{A}_{n}\left(X_{H_{3 k}}\right)^{c}\right) \leq e^{-c n^{\beta}} .
$$

Proof. Let $\alpha>0$ and set $r=n^{\alpha}$. Set $\overline{\mathbb{P}}=\mathbb{P} \times P_{\omega}^{o}$. By construction, the events $\mathcal{A}_{n}\left(X_{H_{3 k}}\right)$ are $\overline{\mathbb{P}}$-independent, and we have for every $k$,

$$
\overline{\mathbb{P}}\left(\mathcal{A}_{n}\left(X_{H_{3 k}}\right)\right) \geq \mathbb{P}\left(\mathcal{A}_{n}\right) \geq \mathbb{P}\left(\omega_{e} \geq 1 / 2\right) \mathbb{P}\left(\omega_{e} \in[1 / n, 2 / n]\right)^{4 d-2} .
$$

Therefore, it comes that for $n$ large enough

$$
\overline{\mathbb{P}}\left(\bigcap_{k=0}^{r / 3-3} \mathcal{A}_{n}\left(X_{H_{3 k}}\right)^{c}\right) \leq \exp \left(-c n^{\alpha} \mathbb{P}\left(\omega_{e} \in[1 / n, 2 / n]\right)^{4 d-2}\right) .
$$

Then set $p(\omega)=P_{\omega}^{o}\left(\bigcap_{k=0}^{r / 3-3} \mathcal{A}_{n}\left(X_{H_{3 k}}\right)^{c}\right)$ and observe that for positive $c^{\prime}, \beta$ that we choose later, Chebychev inequality yields

$$
\mathbb{P}\left(\omega: p(\omega)>e^{-c^{\prime} n^{\beta}}\right) \leq e^{c^{\prime} n^{\beta}} \mathbb{E}(p(\omega)) \leq \exp \left(c^{\prime} n^{\beta}-c n^{\alpha} \mathbb{P}\left(\omega_{e} \in[1 / n, 2 / n]\right)^{4 d-2}\right) .
$$

Thus the claim follows by Borel-Cantelli lemma if (C) holds and we choose in the last expression $c^{\prime}=c / 2$ and

$$
\beta=\alpha-(4 d-2) \lim _{u \rightarrow 0} \frac{\log \mathbb{P}\left(\omega_{b} \in[u, 2 u]\right)}{\log u} .
$$

Let $r \geq 3$ and define $K$ as the first rank such that $\mathcal{A}_{n}\left(H_{3 k}\right)$ happens, i.e.

$$
K=\inf \left\{k \in\{0, \ldots, r / 3-1\}: \mathcal{A}_{n}\left(X_{H_{3 k}}\right)\right\} ; \quad \text { with } \inf \emptyset=\infty .
$$

Lemma 2.4 Let $\alpha \in(0,1 / 2)$ and $r=n^{\alpha}$. Then $\mathbb{P}_{o}$-a.e. $\omega$, there exists $c=c(d, \xi)>0$ such that for $n$ large enough, we have

$$
P_{\omega}^{o}\left(X_{n} \in B_{3(K+1)} \backslash B_{3 K}\right) \geq c n^{-1}
$$

Proof. Let $\{y, z\}$ be the strong edge of the trap $\mathcal{A}_{n}$ and define the events

$$
D_{n}=\cap_{j=1}^{n}\left\{X_{j} \in\{y, z\}\right\} .
$$

Note that $P_{\omega}^{o}\left(D_{n}\right) \geq(1-(4 d-2) / n)^{n} \geq e^{-(4 d-2)} / 2$. 
If the walk crosses the edge of a trap $\mathcal{A}_{n}$ with conductance larger than $n^{-1}$, it will cost a probability larger than $1 /(2 d n)$. Then the Markov property and Chebychev inequality give the following

$$
\begin{aligned}
P_{\omega}^{o}\left(X_{n} \in B_{3(K+1)} \backslash B_{3 K}\right) & \geq E_{\omega}^{o}\left(\mathbb{1}_{\left\{H_{3 K}<n\right\}} P_{\omega}^{X_{H_{3 K}}}\left(X_{H_{3 K}+1}=y\right) P_{\omega}^{y}\left(D_{n}\right) ; K<\infty\right) \\
& \geq \frac{e^{-(4 d-2)} / 2}{2 d} n^{-1} P_{\omega}^{o}\left(H_{3 K}<n, K<\infty\right) \\
& =\frac{e^{-(4 d-2)} / 2}{2 d} n^{-1}\left(P_{\omega}^{o}(K<\infty)-n^{-1} E_{\omega}^{o}\left(H_{3 K} ; K<\infty\right)\right)
\end{aligned}
$$

On the one hand, remark that by Lemma 2.3, $P_{\omega}^{o}(K<\infty)$ tends to 1 . On the other hand, from [4, Lemma 4.1] and Borel-Cantelli, for $n$ large enough

$$
\sup _{x \in \mathscr{C}_{\xi} \cap B_{n} \alpha}\left|\mathscr{G}_{x}\right| \leq(\log n)^{2 d} .
$$

Then Lemma 2.2 yields that for $\xi$ small enough and $n$ large enough

$$
n^{-1} E_{\omega}^{o}\left(H_{3 K} ; K<\infty\right) \leq n^{-1} E_{\omega}^{o}\left(H_{r}\right) \leq c(\log n)^{2 d} n^{2 \alpha-1}
$$

which tends to 0 for $\alpha<1 / 2$. And the claim follows.

We are now ready for the proof of Theorem.

Proof of Theorem 1.1. Let $\alpha \in(0,1 / 2)$. Set $r=n^{\alpha}$ and $B_{k}^{\circ}=B_{3(k+1)} \backslash B_{3 k}$. Now observe that by the Markov property and reversibility, we have

$$
\mathrm{P}_{\omega}^{2 n}(o, o) \geq \sum_{k=0}^{r / 3-1} \sum_{x \in B_{k}^{\circ}} \mathrm{P}_{\omega}^{n}(o, x) \mathrm{P}_{\omega}^{n}(x, o) \geq \sum_{k=0}^{r / 3-1} \sum_{x \in B_{k}^{\circ}} \mathrm{P}_{\omega}^{n}(o, x)^{2} \frac{\pi(o)}{\pi(x)}
$$

Bounding $\pi(x) \leq 2 d$ and using Cauchy-Schwarz, we get

$$
\mathrm{P}_{\omega}^{2 n}(o, o) \geq \frac{\pi(o)}{2 d} \sum_{k=0}^{r / 3-1}\left|B_{k}^{\circ}\right|^{-1} P_{\omega}^{o}\left(X_{n} \in B_{k}^{\circ}\right)^{2} .
$$

Since for all $k \in\{0, \ldots, r / 3-1\},\left|B_{k}^{\circ}\right| \leq c n^{\alpha(d-1)}$, and using $K$, we obtain that

$$
\text { the r.h.s. of }(2.17) \geq c \pi(o) n^{-\alpha(d-1)} P_{\omega}^{o}\left(X_{n} \in B_{K}^{\circ}\right)^{2} .
$$

Then Lemma 2.4 yields the result.

\section{ACKNOWLEDGEMENT}

My sincere thanks to Nina, Pierre and Youcef Bey.

\section{REFERENCES}

[1] N. Berger, M. Biskup, C. E. Hoffman, G. Kozma, Anomalous heat-kernel decay for random walk among bounded random conductances, Ann. Inst. Henri Poincaré Probab. Stat. 44 (2008), no. 2, 374-392. 
[2] M. Biskup and O. Boukhadra, Subdiffusive heat-kernel decay in four-dimensional i.i.d. random conductance models, J. London Math. Soc. 86 (2012), no. 2, 455-481.

[3] O. Boukhadra, Heat-kernel estimates for random walk among random conductances with heavy tail, Stochastic Process. Appl. 120 (2010), no. 2, 182-194.

[4] O. Boukhadra T. Kumagai and P. Mathieu, Local CLT for the polynomial lower tail RCM, J. Math. Soc. Japan Vol. 67, No. 4 (2015) pp. 1413-1448.

[5] L. R. G. Fontes and P. Mathieu, On symmetric random walks with random conductances on $\mathbb{Z}^{d}$, Probab. Theory Related Fields 134 (2006), no. 4, 565-602.

[6] G. Grimmett, Percolation, second edition, Grundlehren der Mathematischen Wissenschaften, 321, Springer, Berlin, 1999.

[7] T. Kumagai, Random Walks on Disordered Media and their Scaling Limits, Lect. Notes in Math., 2101, École d'Été de Probabilités de Saint-Flour XL-2010. Springer, New York, (2014). 